\title{
Complex Fuzzy Structured Element and Its Properties
}

\author{
Kaiqi ZOU ${ }^{1}$, Shigang LIU ${ }^{1}$, Xiaoyue ZHOU ${ }^{1,2}$ \\ ${ }^{1}$ Information Engineering College, Dalian University, Dalian China \\ ${ }^{2}$ Management College, China University of Mining \&Technology, Beijing, China \\ Email: zoukq@vip.sina.com,liushigang103@163.com
}

\begin{abstract}
The first part of this paper gives the definition about complex fuzzy structured element on the basis of one-dimensional fuzzy structured element and some of its property. The following part introduces its limit and continuity. All of this has opened up a vision for the research of fuzzy structured element, and also played an important role in promoting its progress.
\end{abstract}

Keywords: complex fuzzy structured element, $E^{(2)}-F^{(2)}$ modulus, continuity of complex fuzzy structured element

\section{Introduction}

Many scholars have further studied the fuzzy structured element, and they also have given out a lot of properties about the fuzzy structured element. But the study of complex fuzzy structured element is at its beginning stage. In this paper, we study the complex fuzzy structured element, which breaking the blank situation of researching the complex fuzzy structured element, and laying a foundation for the further research of complex fuzzy structured element. It also breaks the blank field of researching area in complex fuzzy number, and brings motivations for people to study the calculus in the field of the fuzzy structured element. Meanwhile, it also enriches the knowledge of fuzzy mathematics and widens the vision for the research of the fuzzy control, fuzzy decision-making, etc. It also broadens a new area for its study. Of course, the study of this paper is a qualitative leap from the real area to the complex area, and brings great convenience for the information classification and management. In order to give a clear introduction for the following part, we give the following illustrations as the starting point, for a coordinate point $f(a, b)=a+i b$ of two-dimensional space, every coordinate point $f(x, y)=$ $x+i y$ its degree of near $f(a, b)$ can be expressed by the fuzzy sets in fuzzy numbers, Recorded as $A^{(2)}(x, y)$ (hear we add (2) in the upper right corner of the two-dimensional space of symbols, which is different from one-

\footnotetext{
*This paper is supported by the National Natural Science Foundation of China (Grant No.60873042) and Liaoning Provincial Department of Education(Grant No.2008024)
}

dimensional space of symbols). In one-dimensional space we have membership function $B(x)=e^{-\left(\frac{x-a}{\sigma}\right)^{2}}$ (of course there are others). As to the membership function of two-dimensional space, we take the membership function $B(x)=e^{-\left(\frac{x-a}{\sigma}\right)^{2}}$ and $C(y)=e^{-\left(\frac{y-b}{\sigma}\right)^{2}}$, according we defined the membership function of two-dimensional space as $\underset{\sim}{A^{(2)}}(x, y)=\frac{B(x)+C(y)}{2}$.

\section{The $E^{(2)}-F^{(2)}$ Modulus of the Complex}

\section{Fuzzy Structured Element and Its Related Concept}

\subsection{The Complex Fuzzy Structured Element}

Definition 2.1.1 Let $E^{(2)}$ be a fuzzy set of $R^{2}$ in the two-dimensional real number field, its membership function recorded as: $\underset{\sim}{E^{(2)}}(x, y)$, here $\underset{\sim}{E^{(2)}}(x, y)=\frac{B(x)+C(y)}{2}$, $x, y \in R$, if $B(x), C(y)$ meet the following nature, we say the two-dimensional fuzzy set $E^{(2)}$ is complex fuzzy structuring element of $R^{2}$.

1) $B(0)=1, C(0)=1, B(1+0)=B(-1-0)=0, C(1+0)=$ $C(-1-0)=0$

2) In the interval $[-1,0) \quad B(x), \quad C(y)$ is a single by 
left-continuous function, In the interval $(0,1]$ is a single down the right continuous function.

3) When $-\infty<x<-1,-\infty<y<-1$ and $1<x<\infty$, $1<y<\infty$, we have $B(x)=C(y)=0$, that is to say $E^{(2)}(x, y)=0$.

If a fuzzy number $A^{(2)}=a+r E^{(2)}+i t F^{(2)}$ (here $r, t$ are real numbers, and $r, t>0, E^{(2)}, F^{(2)}$ are complex fuzzy structuring element), we say $A^{(2)}$ is generated by a linear of $E^{(2)}, F^{(2)}$, here $A^{(2)}(x, y)=$ $\frac{\left(a+r{\underset{\sim}{E}}^{(2)}(x, y)\right)+t F_{\sim}^{(2)}(x, y)}{2}$. Here we state $\varepsilon\left(\underset{\sim}{\left.E^{(2)}-F_{\sim}^{(2)}\right)}\right.$ is all the fuzzy numbers generated by the linear $E^{(2)}$ and $F^{(2)}$.

Definition 2.1.2 $\varepsilon\left(E^{(2)}-F^{(2)}\right)$ is all the fuzzy numbers generated by the linear $E^{(2)}, F^{(2)}$, let $A^{(2)} \in \varepsilon\left(E^{(2)}-F^{(2)}\right)$. There exist limited real numbers $a, r, t \in R, r, t>0$, such that $A^{(2)}=a+r E^{(2)}+i t F^{(2)}$, $r, t>0$, we say $\sqrt{a^{2}+r^{2}+t^{2}}$ is the $E^{(2)}-F^{(2)}-$ modulus of the complex fuzzy number $A^{(2)}$. Recorded as $|\underset{\sim}{A}|_{E^{(2)}-F^{(2)}}$, That is

$$
|A|_{E^{(2)}-F^{(2)}}=\sqrt{a^{2}+r^{2}+t^{2}}
$$

\section{Definition 2.1.3 $\quad E^{(2)}-F^{(2)} \quad$-distance}

$\left(E-d i s \tan c e^{[7]}\right)$, let complex fuzzy number $A_{\sim}^{(2)}, B^{(2)} \in \varepsilon\left(E_{\sim}^{(2)}-F^{(2)}\right)$ and $A^{(2)}=a_{1}+r_{1} E^{(2)}+i t_{1} F_{\sim}^{(2)}$, $B^{(2)}=a_{2}+r_{2} E_{\sim}^{(2)}+i t_{2} F^{(2)}$, here $a_{1}, a_{2}, r_{1}, r_{2}, t_{1}, t_{2} \in R$ and $r_{1}, r_{2}, t_{1}, t_{2}>0$.

We say $\sqrt{\left(a_{1}-a_{2}\right)^{2}+\left(r_{1}-r_{2}\right)^{2}+\left(t_{1}-t_{2}\right)^{2}}$ is the $E^{(2)}-F^{(2)}$-distance of the complex fuzzy number $A_{\sim}^{(2)}$ and $B_{\sim}^{(2)}$, Recorded as $d_{E^{(2)}-F^{(2)}}\left({\underset{\sim}{(2)}}^{(2)} \boldsymbol{\sim}^{(2)}\right)$, That is

$$
d_{E_{\sim}^{(2)}-F_{\sim}^{(2)}}\left(A_{\sim}^{(2)}, B_{\sim}^{(2)}\right)=\sqrt{\left(a_{1}-a_{2}\right)^{2}+\left(r_{1}-r_{2}\right)^{2}+\left(t_{1}-t_{2}\right)^{2}}
$$

\subsection{Subtraction and Multiply of Complex Fuzzy Structuring Element}

Definition 2.2.1. Let $A^{(2)}, B^{(2)}$, $C^{(2)} \in \varepsilon\left(E^{(2)}-F^{(2)}\right), \quad A^{(2)}=a_{1}+r_{1} E^{(2)}+i t_{1} F^{(2)}$, $B^{(2)}=a_{2}+r_{2} E^{(2)}+i t_{2} F^{(2)}, \quad C^{(2)}=a_{3}+r_{3} E^{(2)}+i t_{3} F^{(2)}$, here $a_{1}, a_{2}, r_{1}, r_{2}, t_{1}, t_{2} \in R$ and $r_{1}, r_{2}, t_{1}, t_{2}>0$. If $a_{3}=a_{1}+a_{2}$, $r_{3}=r_{1}+r_{2}, t_{3}=t_{1}+t_{2}$, we say $C^{(2)}$ is $A^{(2)}$ plus $B^{(2)}$. Recorded as $C^{(2)}=A^{(2)}+B^{(2)}$.

Definition 2.2.2 Let $A_{\sim}^{(2)}, B_{\sim}^{(2)}, C^{(2)} \in \varepsilon\left(E_{\sim}^{(2)}-F^{(2)}\right)$,

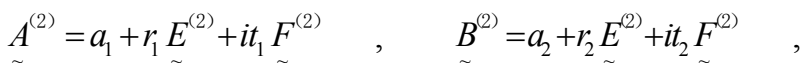
$C^{(2)}=a_{3}+r_{3} E^{(2)}+i t_{3} F^{(2)}$, here $a_{1}, a_{2}, r_{1}, r_{2}, t_{1}, t_{2} \in R$ and $r_{1}, r_{2}, t_{1}, t_{2}>0$. If $a_{3}=a_{1}-a_{2}, r_{3}=r_{1}-r_{2}, t_{3}=t_{1}-t_{2}$, we say $C^{(2)}$ is the subtraction of $A^{(2)}$ and $B^{(2)}$, Recorded as $C^{(2)}=A^{(2)}-B^{(2)}$.

Definition 2.2.3 Let $A^{(2)}, C^{(2)} \in \varepsilon\left(E^{(2)}-F^{(2)}\right)$, $A^{(2)}=a_{1}+r_{1} E^{(2)}+i t_{1} F^{(2)}, \quad C^{(2)}=a_{3}+r_{3} E^{(2)}+i t_{3} F^{(2)}$, here $a_{1}, a_{3}, r_{1}, r_{3}, t_{1}, t_{3} \in R$ and $r_{1}, r_{3}, t_{1}, t_{3}>0$. If $a_{3}=k a_{1}$, $r_{3}=k r_{1}, t_{3}=k t_{1}$, we say $C^{(2)}$ is $k$ multiply $A^{(2)}$. Recorded as $C^{(2)}=k A^{(2)}$.

\section{The Limit of the Complex Fuzzy Structuring Element}

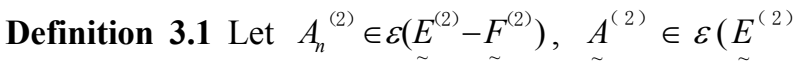
$\left.-F^{(2)}\right)$, if $\forall \varepsilon>0, \exists N$, such that $n>N$, $d_{E_{\sim}^{(2)}-F_{\sim}^{(2)}}\left(A_{\sim}{ }_{\sim}^{(2)}, A_{\sim}^{(2)}\right)<\varepsilon$ is true, we say $\left\{\underset{\sim}{A_{n}{ }^{(2)}}\right\}$ converge to $\underset{\sim}{A^{(2)}}$ according to fuzzy distance $d_{E^{(2)}-F^{(2)}}$.

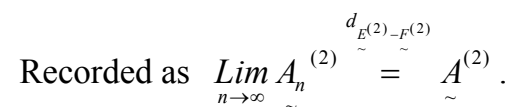

In the following we give a theorem about the convergence of complex fuzzy numbers with the help of $E^{(2)}-F^{(2)}$-distance. 
Theorem 3.1. Let $A_{n}^{(2)}, \underset{\sim}{A^{(2)}} \in \mathcal{E} \underset{\sim}{\left(E^{(2)}\right)}, \underset{\sim}{A^{(2)}}=$

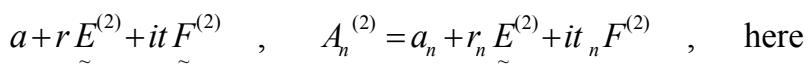
$a, a_{n}, r, r_{n}, t, t_{n} \in R \quad$ and $\quad r, r_{n}, t, t_{n}>0 \quad$, if $\forall \varepsilon>0, \exists \delta>0 \quad, \quad$ such that when $\left|a-a_{n}\right|<\delta$, $\left|r-r_{n}\right|<\delta,\left|t-t_{n}\right|<\delta$, constant have inequality $d_{E^{(2)}-F^{(2)}}$ $\left(A_{n}{ }^{(2)}, A^{(2)}\right)<\varepsilon$ is true. That is $A_{n}{ }^{(2)}$ converge to $\stackrel{\sim}{\sim}^{(2)}$ according to fuzzy distance $d_{E^{(2)}-F^{(2)}}$.

Proof. In fact, we choose $\delta=\frac{\varepsilon}{\sqrt{3}}$,

From the meaning of the question we know $\exists N$, when $n .>N, \quad\left|a-a_{n}\right|<\frac{\varepsilon}{\sqrt{3}}, \quad\left|r-r_{n}\right|<\frac{\varepsilon}{\sqrt{3}}$, $\left|t-t_{n}\right|<\frac{\varepsilon}{\sqrt{3}}$, that is when $n .>N$,

$$
\begin{aligned}
d_{E_{\sim}^{(2)}-F_{\sim}^{(2)}}\left(A_{\sim}^{\left(A_{n}\right.}, \underset{\sim}{A}{ }^{(2)}\right)= & \sqrt{\left(a-a_{n}\right)^{2}+\left(r-r_{n}\right)^{2}+\left(t-t_{n}\right)^{2}} \\
& <\sqrt{\left.\left(\frac{\varepsilon}{\sqrt{3}}\right)^{2}+\left(\frac{\varepsilon}{\sqrt{3}}\right)^{2}+\left(\frac{\varepsilon}{\sqrt{3}}\right)^{2}\right]=\varepsilon}
\end{aligned}
$$

So $A_{n}^{(2)}$ converge to $A^{(2)}$ according to the fuzzy distance $d_{E^{(2)}-F^{(2)}}$

Theorem 3.2 (Complex fuzzy limits with a number of addition, subtraction and multiply theorem)

$$
\begin{aligned}
& \text { Let }\left\{\begin{array}{c}
A_{\sim}^{(2)} \\
\sim
\end{array}, \quad\left\{\underset{\sim}{B_{n}^{(2)}}\right\} \in \underset{\sim}{E}(\underbrace{(2)}_{\sim}-F_{\sim}^{(2)}), \quad \underset{\sim}{A^{(2)}},{\underset{\sim}{\sim}}^{(2)} \in\right.
\end{aligned}
$$

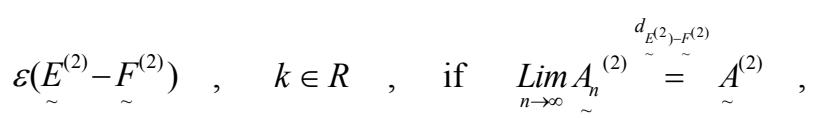

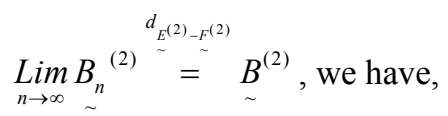

$$
\begin{aligned}
& \text { 1) } \operatorname{Lim}_{n \rightarrow \infty}\left(A_{\sim}^{(2)}+B_{n}^{(2)}\right) \stackrel{d_{E^{(2)}-F^{(2)}}^{\sim}}{=} A_{\sim}^{(2)}+B_{\sim}^{(2)} \\
& \text { 2) } \operatorname{Lim}_{n \rightarrow \infty}\left(A_{n}^{(2)}-B_{n}^{(2)}\right) \stackrel{\substack{d^{(2)}-F^{(2)} \\
\sim}}{=} \underset{\sim}{(2)}-B_{\sim}^{(2)} \\
& \text { 3) } \operatorname{Lim}_{n \rightarrow \infty} A_{n}^{(2)} \stackrel{d_{E^{(2)}-F^{(2)}}^{\sim}}{=} k \underset{\sim}{\sim} A^{(2)}
\end{aligned}
$$

Proof. Only serves to prove (3), It is easy to get the result when $k=0$. In the following we prove the conclusion is correct when $k \neq 0$.

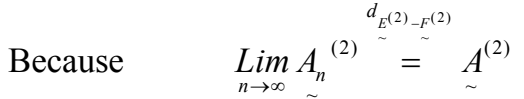

$$
\begin{aligned}
& \text { Then we know } \forall \varepsilon>0, \exists N, n>N, \\
& d_{E_{\sim}^{(2)}-F_{\sim}^{(2)}}\left(A_{\sim}^{(2)}, A_{\sim}^{(2)}\right)<\frac{\varepsilon}{k} \quad(k \neq 0)
\end{aligned}
$$

So

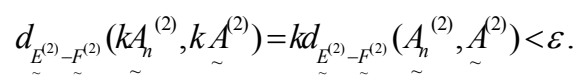

Theorem 3.3 (The uniqueness of the limit) Let $\left.\left.\left\{{\underset{\sim}{\sim}}^{(2)}\right\} \in \varepsilon \underset{\sim}{E^{(2)}}\right), \quad \underset{\sim}{A^{(2)}},{\underset{\sim}{B}}^{(2)} \in \varepsilon \underset{\sim}{E^{(2)}}\right), \quad$ if

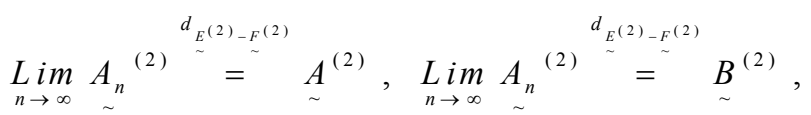
we have $A^{(2)}=B^{(2)}$.

Proof. Because

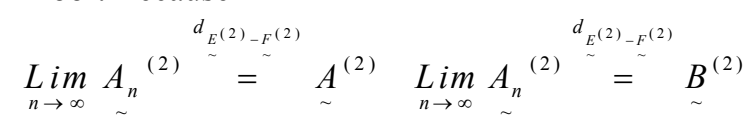

So

$\forall \varepsilon>0, \exists N_{1}, N_{2}>0$, when $n>N_{1}$, we have $d_{E_{\sim}^{(2)}-F_{\sim}^{(2)}}\left(A_{\sim}^{(2)}, \underset{\sim}{A^{(2)}}\right)<\frac{\varepsilon}{2}$

Similarly, when $n>N_{2}$, we have $d_{E^{(2)}-F^{(2)}}\left(A_{n}{ }^{(2)}, B_{\sim}^{(2)}\right)$ $<\frac{\varepsilon}{2}$

Let $N=\max \left(N_{1}, N_{2}\right)$, When $n>N$, then $\left.0<d_{E^{(2)}-F^{(2)}}\left(\underset{\sim}{(2)}, \sim_{\sim}^{(2)}\right)<d_{E^{(2)}-F^{(2)}} \underset{\sim}{A^{(2)}}, A_{n}^{(2)}\right)+d_{E^{(2)}-F^{(2)}}\left(A_{n}^{(2)},{\underset{\sim}{(2)}}^{(2)}\right)<\varepsilon$ As $\varepsilon$ is arbitrary we obtain:

$$
d_{E_{\sim}^{(2)}-F_{\sim}^{(2)}}\left(A_{\sim}^{(2)}, B_{\sim}^{(2)}\right)=0
$$

That is

$$
\underset{\sim}{A}{ }^{(2)}=\underset{\sim}{B^{(2)}}
$$

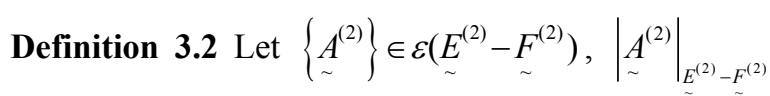
$=\sqrt{a_{2}+r_{2}+t_{2}}$, if $\exists M>0, M \in R, \quad\left|A_{\sim}^{(2)}\right|_{E_{\sim}^{(2)}-F_{\sim}^{(2)}} \leq M \quad$ is always right, we say $A^{(2)}$ is bounded.

Theorem 3.4 (Bounded theorem) Let $\left\{{\underset{\sim}{A_{n}}}^{(2)}\right\} \in \mathcal{E}\left(\underset{\sim}{E^{(2)}}\right), \quad \underset{\sim}{A^{(2)}} \in \mathcal{E}\left(\underset{\sim}{E^{(2)}}\right), \quad$ if $\operatorname{Lim}_{n \rightarrow \infty}{\underset{\sim}{\sim}}^{(2)}$ $\stackrel{d^{(2)}-F^{(2)}}{\sim}=A_{\sim}^{(2)}$ and $\underset{\sim}{A^{(2)}}$ is bounded, then we say $\left\{\begin{array}{c}A_{n}{ }^{(2)} \\ \sim\end{array}\right\}$ 
is bounded.

Proof. because $A^{(2)}$ is bounded and $d_{E^{(2)}-F^{(2)}}$

$\operatorname{Lim}_{n \rightarrow \infty} A_{n} \stackrel{(2) \stackrel{E^{(2)}-F^{(2)}}{\sim}}{=} A_{\sim}^{(2)}$, then $\exists M_{1}$, s.t $\left|\underset{\sim}{A}{ }^{(2)}\right|<M_{1}$, also

$\exists N$ when $n>N$, we have $d_{E^{(2)}-F^{(2)}}\left(\underset{\sim}{A^{(2)}}, A_{n}^{(2)}\right)<\varepsilon$,

Let $\varepsilon=1$, then we have $\left\|A^{(2)}|-| A_{n}^{(2)}\right\|<1$

That is to say $\left|A_{n}^{(2)}\right|<\left|A^{(2)}\right|+1$

Here we choose $M=M_{1}+1$

We can get $\left|A_{n}^{(2)}\right| \leq M$, So $\left\{A_{n}^{(2)}\right\}$ is bounded

\section{The Continuous Definition of Complex Fuzzy Structuring Element}

First of all, we know $a+r E^{(2)}$ is a fuzzy structuring element, for $A^{(2)} \in \varepsilon\left(E^{(2)}\right), \quad A^{(2)}=a+r E^{(2)}+i t F^{(2)}$, $r, t>0$, refer to the continuity of fuzzy structuring element, we give out the continuous definition of complex fuzzy structuring element.

Definition 4.1 Let $A^{(2)}=a+r E^{(2)}+i t F^{(2)}, r$, $t \in R$ and $r, t>0$, if fuzzy structuring element $a+r E^{(2)}$ and $t F^{(2)}$ is continuous at every point $(x, y) \in D \times D \subset R \times R$, we say $A^{(2)}$ is continuous in $D \times D$.

Now we give the continuous definition of complex fuzzy structuring element at some point.

Definition 4.2 Let $A^{(2)} \in \varepsilon\left(E^{(2)}\right),\left(x_{0}, y_{0}\right) \in R \times R$, if $d_{E^{(2)}-F^{(2)}}$

$\operatorname{Lim}_{x \rightarrow x_{0} \sim} A^{(2)}(x, y) \stackrel{\sim}{=} A_{0}{ }^{(2)}\left(x_{0}, y_{0}\right)$, we say the complex $x \rightarrow x_{0}$
$y \rightarrow y_{0}$

fuzzy structuring element $A^{(2)}$ is continuous at $\left(x_{0}, y_{0}\right)$.

In the above definition, if we want to determine whether a fuzzy-valued functions is continuous in $D \times D$, we should verify every point $(x, y) \in D \times D$, which is very different to verification. Therefore, this is only a theory definition. Here we give a determine theorem about the continuous of complex fuzzy structuring element.

Determine theorem 4.1 Let $A^{(2)}=a+r E^{(2)}+i t F^{(2)}, r$, $t \in R \quad$ and $\quad r, t>0, \quad \underset{\sim}{E^{(2)}}(x, y)=\frac{B_{E}(x)+C_{E}(y)}{2}$,
$F_{\sim}^{(2)}(x, y)=\frac{B_{F}(x)+C_{F}(y)}{2}, \forall(x, y) \in R$, then as long as the membership function $B_{E}(x), C_{E}(y), B_{F}(x)$, $C_{F}(y)$ is continuous in $D$, we obtain $A^{(2)}(x, y)$ is continuous in $D$.

Proof. From the preceding introduce we know that:

$$
A_{\sim}^{(2)}(x, y)=\frac{\left(a+r \sim_{\sim}^{E} E^{(2)}(x, y)\right)+t \underset{\sim}{F_{\sim}^{(2)}}(x, y)}{2}
$$

Therefore $\forall\left(x_{0}, y_{0}\right) \in D \times D$ we have

$$
{\underset{\sim}{\sim}}^{(2)}\left(x_{0}, y_{0}\right)=\frac{\left(a+r \underset{\sim}{E^{(2)}}\left(x_{0}, y_{0}\right)\right)+t \underset{\sim}{F^{(2)}}\left(x_{0}, y_{0}\right)}{2}
$$

So

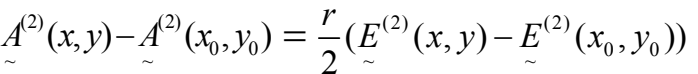

$$
\begin{aligned}
& +\frac{t}{2}\left(F_{\sim}^{(2)}(x, y)-F_{\sim}^{(2)}\left(x_{0}, y_{0}\right)\right) \\
& =\frac{r}{2}\left(\frac{B_{E}(x)}{2}-\frac{B_{E}\left(x_{0}\right)}{2}\right)+\frac{r}{2}\left(\frac{C_{E}(y)}{2}-\frac{C_{E}\left(y_{0}\right)}{2}\right) \\
& +\frac{t}{2}\left(\frac{B_{F}(x)}{2}-\frac{B_{F}\left(x_{0}\right)}{2}\right)+\frac{t}{2}\left(\frac{C_{F}(y)}{2}-\frac{C_{F}\left(y_{0}\right)}{2}\right)
\end{aligned}
$$

Because the membership function $B_{E}(x), C_{E}(y)$, $B_{F}(x), C_{F}(y)$ are continuous in $D$, we know when $\forall \varepsilon>0, \exists \delta>0,\left|x-x_{0}\right|<\delta,\left|y-y_{0}\right|<\delta$,

$$
\begin{aligned}
& \frac{B_{E}(x)}{2}-\frac{B_{E}\left(x_{0}\right)}{2}<\frac{\varepsilon}{2 r}, \frac{C_{E}(y)}{2}-\frac{C_{E}\left(y_{0}\right)}{2}<\frac{\varepsilon}{2 r} \\
& \frac{B_{F}(x)}{2}-\frac{B_{F}\left(x_{0}\right)}{2}<\frac{\varepsilon}{2 t}, \frac{C_{F}(y)}{2}-\frac{C_{F}\left(y_{0}\right)}{2}<\frac{\varepsilon}{2 t}
\end{aligned}
$$

Thus we obtain

$$
A_{\sim}^{(2)}(x, y)-A_{\sim}^{(2)}\left(x_{0}, y_{0}\right)<\varepsilon
$$

Due to the arbitrariness of $(x, y) \in D$, we know $A^{(2)}(x, y)$ is continuous in $D$.

\section{References}

[1] D. Dubois and H. Prade, "Operations on fuzzy numbers," [J] Systems Sci, Vol. 9, No. 6, pp. 613-626, 1978.

[2] G. Zhang, "Fuzzy continuous function and ir's propertyes [J]," Fuzzy Sets and Systems, Vol. 43, pp. 159-171, 1991.

[3] M. Mizumoto, Tanakak, "The four operations for arithmetic on fuzzy numbers [J]," Systems Comput Controls, Vol. 7, No. 5, pp. 73-81, 1976. 
[4] J. J. Buckley, "Fuzzy complex analysis II: Integration [J]," Fuzzy Sets and Systems, Vol. 49, pp. 171-179, 1992.

[5] S. Z. Guo, "Structured element representation of limit and continuity of fuzzy - valued function [J]," Journal of Hainan normal university (nature science), Vol. 21, No. 4, pp. 351-356, 2008.
[6] S. Z. Guo, Z. X. Su, L. Wang, "Method of structured element in fuzzy analysis and calculation [J]," Fuzzy systems and mathematics, Vol. 18, No. 3, pp. 68-75, 2004.

[7] S. Z. Guo, "Method of structuring element in fuzzy analysis ( II ) [J]," Journal of Liaoning Technical University, Vol. 21, No. 6, pp. 808-810, 2002. 